\title{
Fundamental Improvement of a Convergence Test for Iterative Strain-Gage Balance Load Predictions
}

\author{
N. Ulbrich ${ }^{\dagger}$ \\ Jacobs Technology Inc., Moffett Field, California 94035
}

\begin{abstract}
A fundamental improvement of a convergence test for wind tunnel straingage balance load iterations was developed. The improvement became necessary because incorrect test results were obtained whenever the original test was applied to multi-component balances with large load capacity differences. The original test was first published in NASA TN D-6860. It uses an upper bound of the Lipschitz constant to assess convergence characteristics of balance load predictions whenever the Iterative Method is applied. The Lipschitz constant is a function of the partial derivatives of each balance load component with respect to all other load components. Unfortunately, the original definition of the convergence test overlooked the fact that the Lipschitz constant is a dimensionless quantity and that the partial derivative of one load component with respect to another load component is not always dimensionless. Therefore, an improvement of the original test was successfully developed that uses load capacities to make all inputs for the calculation of the Lipschitz constant dimensionless before use. Results from the calibration data analysis of a six-component force balance and a five-component semi-span balance are used to illustrate the application of the improved load iteration convergence test.
\end{abstract}

\section{Nomenclature}

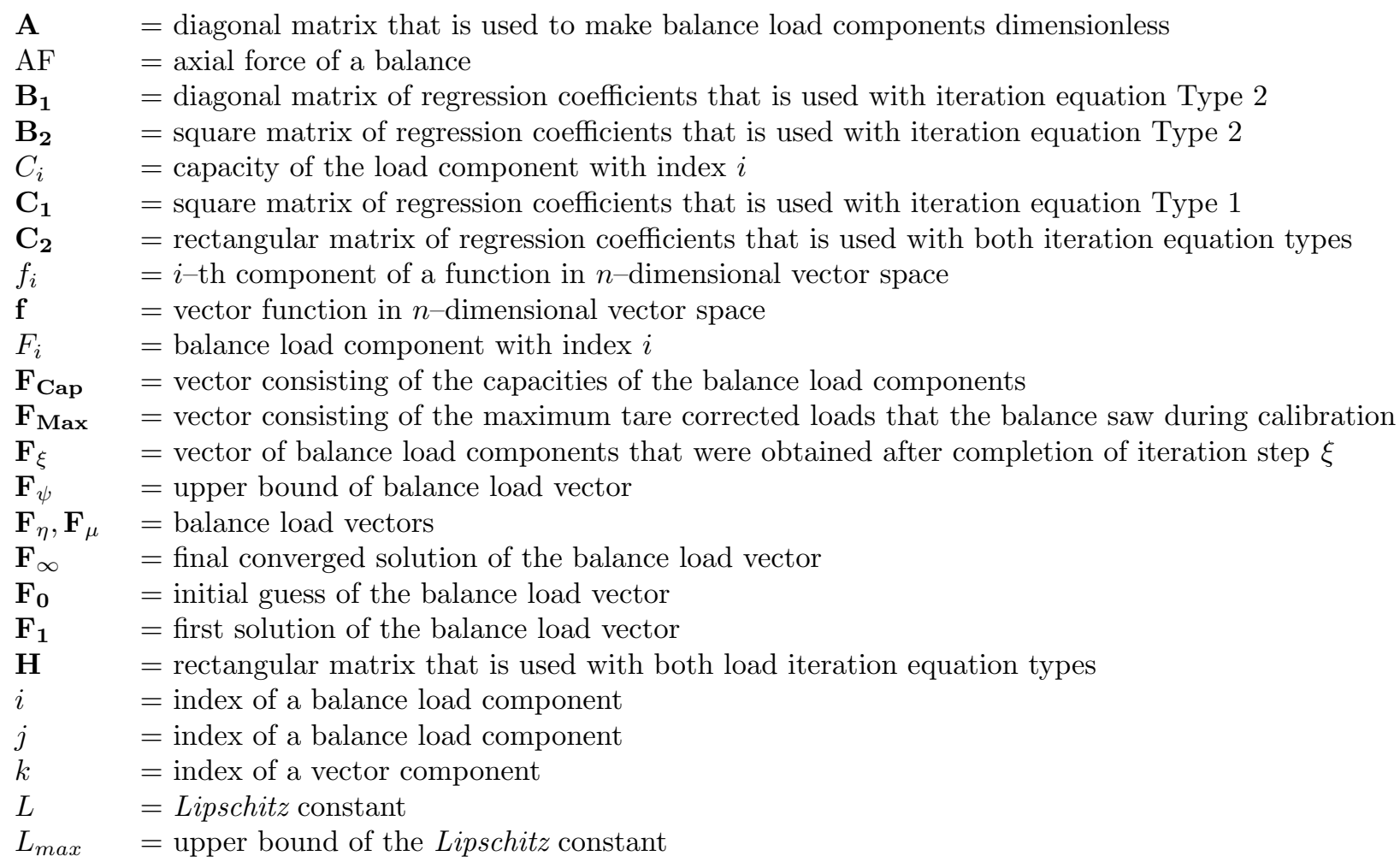

$\dagger$ Aerodynamicist, Jacobs Technology Inc. 


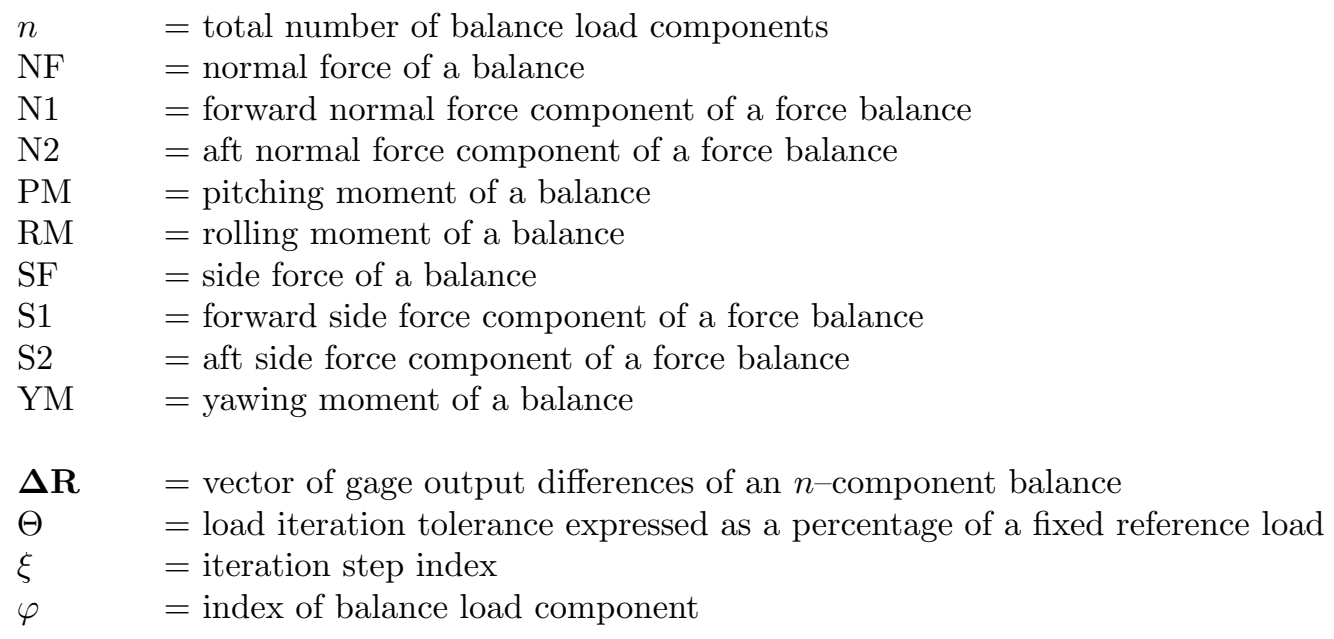

\section{Introduction}

Recently, implementation and use of a convergence test for iterative strain-gage balance load predictions were described in the literature (Ref. [1]). This test can be applied to both types of load iteration equations that are used in the aerospace testing community today. Table 1 below lists these two iteration equation types in matrix format where $\mathbf{F}$ is the load vector, $\boldsymbol{\Delta} \mathbf{R}$ is the vector of output differences, $\xi$ is the iteration step index, and $\mathbf{B}_{\mathbf{1}}, \mathbf{B}_{\mathbf{2}}, \mathbf{C}_{\mathbf{1}}$, and $\mathbf{C}_{\mathbf{2}}$ are the coefficient matrices (see Refs. [2], [3], and [4] for more details).

Table 1: Iteration Equation Types for Strain-Gage Balance Load Prediction.

\begin{tabular}{|c|c|c|}
\hline TYPE & ITERATION EQUATION DEFINITION & $\begin{array}{l}\mathbf{F}_{\mathbf{0}} \equiv \text { INITIAL GUESS } \\
\mathbf{F}_{1} \equiv \text { FIRST SOLUTION }\end{array}$ \\
\hline 1 & $\begin{array}{l}\qquad \mathbf{F}_{\xi}=\mathbf{C}_{\mathbf{1}}^{-1} \boldsymbol{\Delta} \mathbf{R}-\left\{\mathbf{C}_{\mathbf{1}}^{-1} \mathbf{C}_{\mathbf{2}} \cdot \mathbf{H}\left(\mathbf{F}_{\xi-\mathbf{1}}\right)\right\} \\
\ldots \text { taken from Ref. [2], Eq. (3.3.7), or, Ref. [3], Eq. (30) }\end{array}$ & $\begin{array}{l}\mathbf{F}_{\mathbf{0}}=\left[\begin{array}{c}0 \\
\vdots \\
0\end{array}\right] \\
\mathbf{F}_{\mathbf{1}}=\mathbf{C}_{\mathbf{1}}^{-1} \boldsymbol{\Delta} \mathbf{R}\end{array}$ \\
\hline 2 & $\begin{array}{c}\mathbf{F}_{\xi}=\mathbf{B}_{\mathbf{1}}^{-1} \Delta \mathbf{R}-\left\{\mathbf{B}_{\mathbf{1}}^{-1} \mathbf{B}_{\mathbf{2}} \cdot \mathbf{F}_{\xi-\mathbf{1}}+\mathbf{B}_{\mathbf{1}}^{-1} \mathbf{C}_{\mathbf{2}} \cdot \mathbf{H}\left(\mathbf{F}_{\xi-\mathbf{1}}\right)\right\} \\
\ldots \text { taken from Ref. [4], Eq. (26) }\end{array}$ & $\begin{array}{l}\mathbf{F}_{\mathbf{0}}=\left[\begin{array}{c}0 \\
\vdots \\
0\end{array}\right] \\
\mathbf{F}_{\mathbf{1}}=\mathbf{B}_{1}^{-1} \Delta \mathbf{R}\end{array}$ \\
\hline
\end{tabular}

The coefficients of the two iteration equation types above are stored in a "data reduction matrix." It was first shown by Smith in NASA TN D-6860 that these coefficients may be used in combination with an estimate of the upper bound of the load vector of the balance to evaluate the convergence characteristics of the iteration equation within an expected use envelope of the balance (for more detail see Ref. [5]).

NASA TN D-6860 uses the coefficients of the "data reduction matrix" as numerical estimates of the partial derivatives of each balance load component with respect to all other load components. These partial derivatives are needed as input for the calculation of an upper bound of the so-called Lipschitz constant. By design, the Lipschitz constant must be less than one to guarantee convergence of the iteration process that is defined by (i) the chosen iteration equation and (ii) the coefficients of the data reduction matrix. 
In 2018, the author applied the original convergence test of NASA TN D-6860 to data of a multicomponent semi-span balance that had very large load capacity differences. In this case, incorrect iteration convergence test results were obtained. The convergence test predicted iteration divergence even though the observed load iterations of the semi-span balance showed rapid convergence. Further investigations of this "paradox" revealed that the original definition of the convergence test had overlooked the fact that (i) the Lipschitz constant is a dimensionless quantity and that (ii) the partial derivative of one load component with respect to another load component is not always dimensionless. Therefore, a fundamental improvement of the original convergence test was developed that uses load capacities to make all inputs for the calculation of the Lipschitz constant dimensionless before use.

Key elements of the improved load iteration convergence test are described in the next section of the paper. Afterwards, calibration data analysis results of a six-component force balance and a five-component semi-span balance are used to illustrate the application of the improved iteration convergence test.

\section{Description of Improved Convergence Test}

\section{A. Revised Iteration Convergence Test}

It is shown in this section how the original iteration convergence test defined in NASA TN D-6860 was corrected such that it can be applied to all balances independent of the magnitudes of the capacities of their load components. First, the convergence test itself is reviewed. NASA TN D-6860 suggests to use an estimate of the upper bound of the so-called Lipschitz constant for the evaluation of the convergence characteristics of the load iterations (see Ref. [5] for more detail). In general, the report's derivations closely follow steps that are described in Henrici's textbook on numerical analysis. Henrici investigated the convergence characteristics of the following vector "sequence" (see Ref. [6], p. 99, Eq. (95-6)) ...

$$
\mathbf{F}_{\xi}=\mathbf{f}\left(\mathbf{F}_{\xi-1}\right)
$$

where

$$
\left[\mathbf{F}_{\xi}\right]_{n \times 1}=\left[\begin{array}{c}
F_{1} \\
\vdots \\
F_{i} \\
\vdots \\
F_{n}
\end{array}\right]
$$

and where $\xi$ is the "sequence", i.e., iteration step, index. Now, column vector $\mathbf{F}_{\xi}$ can be interpreted as the load vector of an $n$-component balance where $F_{1}$ to $F_{n}$ are the load components of the balance. Similarly, a column vector $\mathbf{F}_{\text {Cap }}$ can be defined whose components $C_{1}$ to $C_{n}$ are the balance load capacities. This vector can be described as follows:

$$
\left[\mathbf{F}_{\mathbf{C a p}}\right]_{n \times 1}=\left[\begin{array}{c}
C_{1} \\
\vdots \\
C_{i} \\
\vdots \\
C_{n}
\end{array}\right]
$$

In addition, the right-hand side of Eq. (1a) may be interpreted as the right-hand side of a load iteration equation that is listed in Table 1 above. Then, we can describe the right-hand side of Eq. (1a) as follows:

$$
\left[\mathbf{f}\left(\mathbf{F}_{\xi-\mathbf{1}}\right)\right]_{n \times 1}=\left[\begin{array}{c}
f_{1}\left(\mathbf{F}_{\xi-\mathbf{1}}\right) \\
\vdots \\
f_{i}\left(\mathbf{F}_{\xi-\mathbf{1}}\right) \\
\vdots \\
f_{n}\left(\mathbf{F}_{\xi-\mathbf{1}}\right)
\end{array}\right]=\left\{\begin{array}{l}
\underbrace{\mathbf{C}_{\mathbf{1}}^{-1} \mathbf{\Delta} \mathbf{R}-\left\{\mathbf{C}_{\mathbf{1}}^{-1} \mathbf{C}_{\mathbf{2}} \cdot \mathbf{H}\left(\mathbf{F}_{\xi-\mathbf{1}}\right)\right\}}_{\text {Iteration Equation Type 1 }} \\
\underbrace{\mathbf{B}_{\mathbf{1}}^{-1} \mathbf{\Delta}-\left\{\mathbf{B}_{\mathbf{1}}^{-1} \mathbf{B}_{\mathbf{2}} \cdot \mathbf{F}_{\xi-\mathbf{1}}+\mathbf{B}_{\mathbf{1}}^{-1} \mathbf{C}_{\mathbf{2}} \cdot \mathbf{H}\left(\mathbf{F}_{\xi-\mathbf{1}}\right)\right\}}_{\text {Iteration Equation Type } 2}
\end{array}\right.
$$


Now, after replacing (i) the left-hand side of Eq. (1a) with the right-hand side of Eq. (1b) and (ii) the right-hand side of Eq. (1a) with the middle term of Eq. (1d), we get:

$$
\underbrace{\left[\begin{array}{c}
F_{1} \\
\vdots \\
F_{i} \\
\vdots \\
F_{n}
\end{array}\right]}_{E q \cdot(1 b)}=\underbrace{\left[\begin{array}{c}
f_{1}\left(\mathbf{F}_{\xi-\mathbf{1}}\right) \\
\vdots \\
f_{i}\left(\mathbf{F}_{\xi-\mathbf{1}}\right) \\
\vdots \\
f_{n}\left(\mathbf{F}_{\xi-\mathbf{1}}\right)
\end{array}\right]}_{E q .(1 d)}
$$

Equation (2) above highlights two important facts: (i) the unit of the component $F_{i}$ must match the unit of the component $f_{i}$ and (ii) the units of the load components $F_{1}$ to $F_{n}$ are not necessarily all the same because the load component of a given balance may be a force or a moment. These two fundamental observations are the key to understanding the subsequent discussion of the error in the original definition of the iteration convergence test that was first described in NASA TN D-6860.

Henrici rigorously showed that the sequence defined in Eq. (1a) will converge to a single value if the Lipschitz constant of the sequence, i.e., the Lipschitz constant of the load iteration process, is less than the threshold of one (see Ref. [6], p. 99-101). This convergence test can be described as follows ...

$$
0 \leq L<1 \Longrightarrow \text { load iteration converges }
$$

where the symbol $L$ represents the Lipschitz constant. In theory, Henrici's convergence test can directly be applied to the load iteration process if a "conservative" upper bound $L_{\max }$ of the Lipschitz constant is obtained from (i) a suitable upper bound $\mathbf{F}_{\psi}$ of the load vector and (ii) upper bounds of the partial derivatives $\partial f_{i}\left(\mathbf{F}_{\psi}\right) / \partial F_{j}$ of the load iteration equations with respect to the individual load components of the balance. Then, the condition for convergence of the load iteration process can be written as follows:

$$
L \leq L_{\max }\left\{\frac{\partial f_{1}\left(\mathbf{F}_{\psi}\right)}{\partial F_{1}}, \frac{\partial f_{1}\left(\mathbf{F}_{\psi}\right)}{\partial F_{2}}, \ldots, \frac{\partial f_{i}\left(\mathbf{F}_{\psi}\right)}{\partial F_{j}}, \ldots, \frac{\partial f_{n}\left(\mathbf{F}_{\psi}\right)}{\partial F_{n}}\right\}<1
$$

It is critical to realize at this point that Henrici's convergence test compares the Lipschitz constant with the dimensionless threshold of one (see right-hand side of inequality ( $3 b)$ above). Therefore, the partial derivatives must be made dimensionless before use so that the resulting estimate of the upper bound $L_{\max }$ of the Lipschitz constant is also dimensionless. Unfortunately, this important step was omitted in the derivation of the load iteration convergence test that is described in NASA TN D-6860. Consequently, Eqs. (8), (B6), and (B8) of NASA TN D-6860 are incorrect as some of the partial derivatives given in those equations could, for example, have a force in the numerator and a moment in the denominator (or vice versa).

Fortunately, it is possible to correct the error that was made in NASA TN D-6860. It is simply required to make the partial derivatives of the balance load components dimensionless before use by introducing the ratio between the capacities of the corresponding balance load pair as a scale factor. Then, the corrected definition of the upper bound of the Lipschitz constant can be expressed as follows: 
IMPROVED DEFINITION OF THE UPPER BOUND OF THE LIPSCHITZ CONSTANT

$$
\begin{gathered}
L \leq L_{\text {max }}=\sqrt{\sum_{i=1}^{n} \sum_{j=1}^{n} \underbrace{\left[\frac{\partial f_{i}\left(\mathbf{F}_{\psi}\right)}{\partial F_{j}} \cdot \frac{C_{j}}{C_{i}}\right]^{2}}_{\text {dimensionless derivative }}}<1 \\
\mathbf{F}_{\psi} \equiv \text { uppere bound of the load vector } \\
\frac{\partial f_{i}\left(\mathbf{F}_{\psi}\right)}{\partial F_{j}} \equiv \text { coefficient of the Jacobian matrix } \mathbf{J}\left\{\mathbf{f}\left(\mathbf{F}_{\psi}\right)\right\} \text { for } \mathbf{F}_{\psi} \\
\frac{C_{j}}{C_{i}} \\
C_{\varphi} \quad \equiv \text { scale factor that makes } \frac{\partial f_{i}\left(\mathbf{F}_{\psi}\right)}{\partial F_{j}} \text { dimensionless }
\end{gathered}
$$

Equation (4) above replaces Eq. (4) in Ref. [1] and Eqs. (8), (B6), and (B8) in Ref. [5]. The upper bound of the partial derivatives is obtained from the Jacobian matrix of the right-hand sides of the load iteration equations that are described in Table 1. Three different choices are suggested in the literature in order to define an upper bound $\mathbf{F}_{\psi}$ of the load vector for the iteration convergence test (see Ref. [5], App. B). In addition, the author suggests to include a fourth choice for the upper bound of the load vector: it is the load vector whose components equal the loads of the calibration data point with longest dimensionless Euclidean length. In theory, the convergence characteristics of this fourth load vector should be closest to the observed convergence behavior of the balance calibration data set that generated the load iteration equation.

The four load vector choices may be a function of the capacities of the balance load components, or, a function of the maximum tare corrected loads that the balance experienced during its calibration, or, a function of the largest value of the square root of the sum of squares of the dimensionless loads of the calibration data points. For convenience, the four load vector options can be summarized as follows:

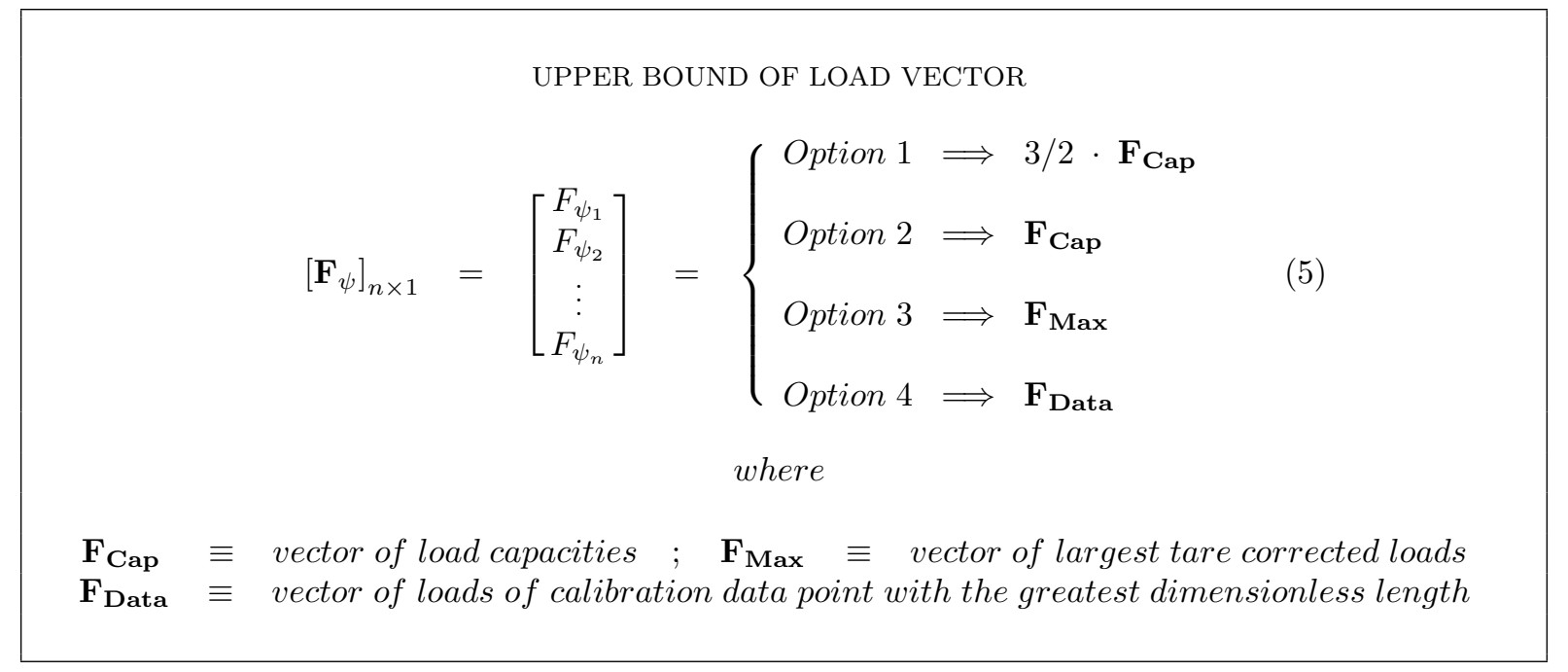

It is important to point out that Option 1, Option 2, and Option 3 above are very "conservative" in nature. They imply that the iteration is performed while all load components are simultaneously loaded.

American Institute of Aeronautics and Astronautics 
This situation rarely exists during the actual use of the balance. Option 4, on the other hand, uses the loads of the applied calibration data point with the largest dimensionless length as input for the calculation of the Lipschitz constant. Therefore, it is the least conservative of the four load vector choices.

The determination of upper bounds of the partial derivatives of the two types of load iteration equations was already described in great detail in Ref. [1]. However, recent experience showed that this definition of the upper bound is often too conservative. Therefore, another improvement was made. Now, only the absolute value of the entire variable part of the load iteration equation is used for the definition of the upper bound. This change can be described as follows ...

$$
\frac{\partial \mathbf{f}\left(\mathbf{F}_{\psi}\right)}{\partial F_{j}} \leq \frac{\partial \mathbf{f}\left(\mathbf{F}_{\psi}\right)}{\partial F_{j}}=\left\{\begin{array}{l}
\underbrace{\frac{\partial}{\partial F_{j}}\left\{\mathbf{C}_{\mathbf{1}}^{-1} \mathbf{C}_{\mathbf{2}} \cdot \mathbf{H}\left(\mathbf{F}_{\psi}\right)\right\} \mid}_{\text {Iteration Equation Type 1 }} \\
\underbrace{\left|\frac{\partial}{\partial F_{j}}\left\{\mathbf{B}_{\mathbf{1}}^{-1} \mathbf{B}_{\mathbf{2}} \cdot \mathbf{F}_{\psi}+\mathbf{B}_{\mathbf{1}}^{-1} \mathbf{C}_{\mathbf{2}} \cdot \mathbf{H}\left(\mathbf{F}_{\psi}\right)\right\}\right|}_{\text {Iteration Equation Type 2 }}
\end{array}\right.
$$

where the operator symbol $|\ldots|$ indicates that the absolute value of each vector component is used (Eq. (6) above replaces Eq. (7) in Ref. [1]). The vector of partial derivatives listed in Eq. (6) above still needs to be computed. The author recommends to compute the derivatives numerically by using the central difference scheme. This approach was described in great detail in Ref. [1].

An improved formula for the lower bound of the number of iterations is derived and discussed in the next section of the paper.

\section{B. Improved Lower Bound of Number Of Iterations}

It was shown in Ref. [1] how a theoretical estimate of the lower bound of the number of load iterations can be computed as a function of (i) the upper bound of the Lipschitz constant, (ii) the given load vector, (iii) the load iteration type, and (iv) the load iteration tolerance. This estimate, i.e., Eq. (9) of Ref. [1], also needs to be corrected in order to take the dimensionless nature of the Lipschitz constant into account.

Again, as shown in Ref. [1], the estimate of the number of iterations can be derived by using a related inequality as a starting point (see also Ref. [6], p. 99, Eq. (5-9)). In our context, this inequality can be written as follows after the balance loads were made dimensionless by using the load capacities ...

$$
\begin{gathered}
\left\|\mathbf{A} \cdot\left(\mathbf{F}_{\xi}-\mathbf{F}_{\infty}\right)\right\| \leq \frac{\left(L_{\max }\right)^{\xi}}{1-L_{\max }} \cdot\left\|\mathbf{A} \cdot\left(\mathbf{F}_{\eta}-\mathbf{F}_{\mu}\right)\right\| \\
{[\mathbf{A}]_{n \times n}=\left[\begin{array}{ccccc}
1 / C_{1} & 0 & 0 & \cdots & 0 \\
0 & 1 / C_{2} & 0 & \cdots & 0 \\
0 & 0 & 1 / C_{3} & \cdots & 0 \\
\vdots & \vdots & \vdots & \vdots & \vdots \\
0 & 0 & 0 & \cdots & 1 / C_{n}
\end{array}\right]}
\end{gathered}
$$

where (i) the exponent $\xi$ is the load iteration step, (ii) $L_{\max }$ is the upper bound of the Lipschitz constant, (iii) $\left\|\mathbf{F}_{\xi}-\mathbf{F}_{\infty}\right\|$ is the Euclidean norm of a load vector difference that is associated with the lower bound of the load change, (iv) $\left\|\mathbf{F}_{\eta}-\mathbf{F}_{\mu}\right\|$ is the Euclidean norm of a load vector difference that is associated with 
the upper bound of the load change, and (v) $\mathbf{A}$ is a square matrix that has the inverse of the capacities of the balance load components on its principle diagonal. Matrix $\mathbf{A}$ is needed to make both sides of inequality (7a) dimensionless. Now, after solving Eq. (7a) for the exponent, i.e., for the load iteration step $\xi$, we get the following inequality that describes the lower bound of the number of required load iterations:

LOWER BOUND OF THE NUMBER OF REQUIRED LOAD ITERATIONS

$$
\xi \geq \frac{\ln \left\{\left(1-L_{\max }\right) \cdot \frac{\left\|\mathbf{A} \cdot\left(\mathbf{F}_{\xi}-\mathbf{F}_{\infty}\right)\right\|}{\left\|\mathbf{A} \cdot\left(\mathbf{F}_{\eta}-\mathbf{F}_{\mu}\right)\right\|}\right\}}{\ln \left\{L_{\max }\right\}}
$$

Equation (9) above replaces Eq. (12) in Ref. [1]. The upper bound $L_{\max }$ of the Lipschitz constant was already defined in Eq. (4). It remains to specify the lower and upper bounds of the load change that are used in Eq. (9) above. The lower bound can be related to the chosen load iteration tolerance $\Theta$ (e.g., $0.0001 \%$ of the upper bound of the loads) and the Euclidean norm of the dimensionless upper bound $\mathbf{A} \cdot \mathbf{F}_{\psi}$ of the load vector $\mathbf{F}_{\psi}$ that is defined in Eq. (5). Then, we get:

$$
\begin{gathered}
\left\|\mathbf{A} \cdot\left(\mathbf{F}_{\xi}-\mathbf{F}_{\infty}\right)\right\| \approx \frac{\Theta}{100} \cdot\left\|\mathbf{A} \cdot \mathbf{F}_{\psi}\right\| \\
\text { where } \\
\Theta \equiv \text { tolerance }=0.0001 \%
\end{gathered}
$$

Similarly, the upper bound can be related to the Euclidean norm of the load change that is obtained by using the upper bound $\mathbf{F}_{\psi}$ of the load vector as input on the right-hand side of Eq. (1d). Consequently, after applying again the square matrix $\mathbf{A}$ such that the right-hand side of Eq. (1d) becomes dimensionless, we get the following estimate of the upper bound:

$$
\left\|\mathbf{A} \cdot\left(\mathbf{F}_{\eta}-\mathbf{F}_{\mu}\right)\right\| \leq\left\{\begin{array}{l}
\underbrace{\left\|\mathbf{A} \cdot\left\{\mathbf{C}_{\mathbf{1}}^{-1} \mathbf{C}_{\mathbf{2}} \cdot \mathbf{H}\left(\mathbf{F}_{\psi}\right)\right\}\right\|}_{\text {Iteration Equation Type } 1} \\
\underbrace{\left\|\mathbf{A} \cdot\left\{\mathbf{B}_{\mathbf{1}}^{-1} \mathbf{B}_{\mathbf{2}} \cdot \mathbf{F}_{\psi}+\mathbf{B}_{\mathbf{1}}^{-1} \mathbf{C}_{\mathbf{2}} \cdot \mathbf{H}\left(\mathbf{F}_{\psi}\right)\right\}\right\|}_{\text {Iteration Equation Type } 2}
\end{array}\right.
$$

Calibration analyis results from (i) the machine calibration of a six-component force balance and (ii) the manual calibration of a five-component semi-span balance are used in the next section to illustrate benefits of the improved load iteration convergence test.

\section{Discussion of Example}

Data from the calibration of two different wind tunnel strain-gage balances is used in this section to illustrate benefits of the improved load iteration convergence test. Each calibration data set was analyzed in two different ways. First, no scaling was applied to the loads, i.e., (i) the scale factor $C_{j} / C_{i}$ was set to one in Eq. (4) and (ii) the square matrix $\mathbf{A}$ was assigned to be the identity matrix. This choice generates test results for the original definition of the convergence test that is described in NASA TN D-6860. Afterwards, the 
improved convergence test was applied to the same balance calibration data set so that differences between original and improved convergence test could be demonstrated.

The first calibration data set comes from a machine calibration of NASA's MC60E balance. The MC60E balance is a six-component force balance that was manufactured by Calspan Force Measurement Systems $(F M S)$ in San Diego. It has a diameter of 2.0 inches and measures five forces and one moment (N1, N2, S1, $\mathrm{S} 2, \mathrm{AF}, \mathrm{RM})$. Table 2 below lists load capacities of the balance:

Table 2: Load capacities of the six-component MC60E force balance.

\begin{tabular}{|c|c|c|c|c|c|c|}
\hline & N1, lbs & N2, lbs & S1, lbs & S2, lbs & RM, in-lbs & AF, lbs \\
\hline \hline Capacity & 2500 & 2500 & 1250 & 1250 & 5000 & 700 \\
\hline
\end{tabular}

The chosen machine calibration data set was obtained in 2016 in FMS' Automatic Balance Calibration System. The final data set consisted of 2091 points that were distributed across 16 load series. Both single and multi-component loadings were applied during the calibration. The calibration data was analyzed using iteration equation Type 1 in combination with a standard 28-term math model that does not use any absolute value or third order terms ( 28 terms $\equiv 27$ terms plus the intercept). Then, the iteration convergence test was applied by using unscaled derivatives for the calculation of the Lipschitz constant. This case matches the original approach that is used in NASA TN D-6860. Figure 1a below shows results of this convergence test for the four possible upper bounds of the load vector.

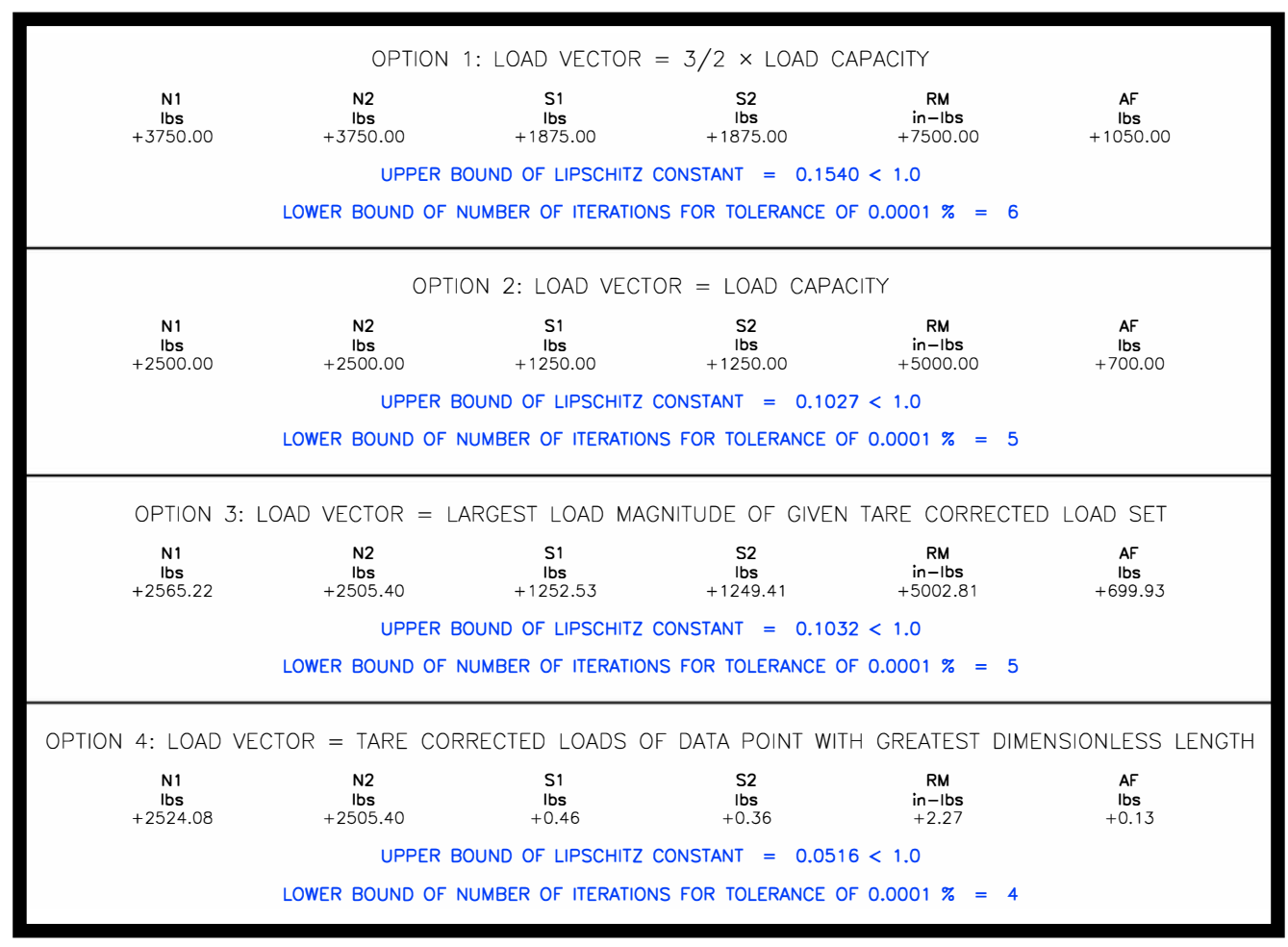

Fig. 1a Convergence test results for the MC60E balance (unscaled derivatives, NASA TN D-6860).

In all cases, the upper bound of the Lipschitz constant is well below the threshold of one. Therefore, load iterations are expected to converge in all four selected load regions that the upper bounds of the load vector define. Now, the iteration convergence test was applied by using scaled derivatives for the calculation of the Lipschitz constant. This choice matches the improved iteration convergence test that is defined in Eqs. (4) and (9) of the current paper. Figure 1b below shows test results for the four possible choices of the upper bound of the load vector. 


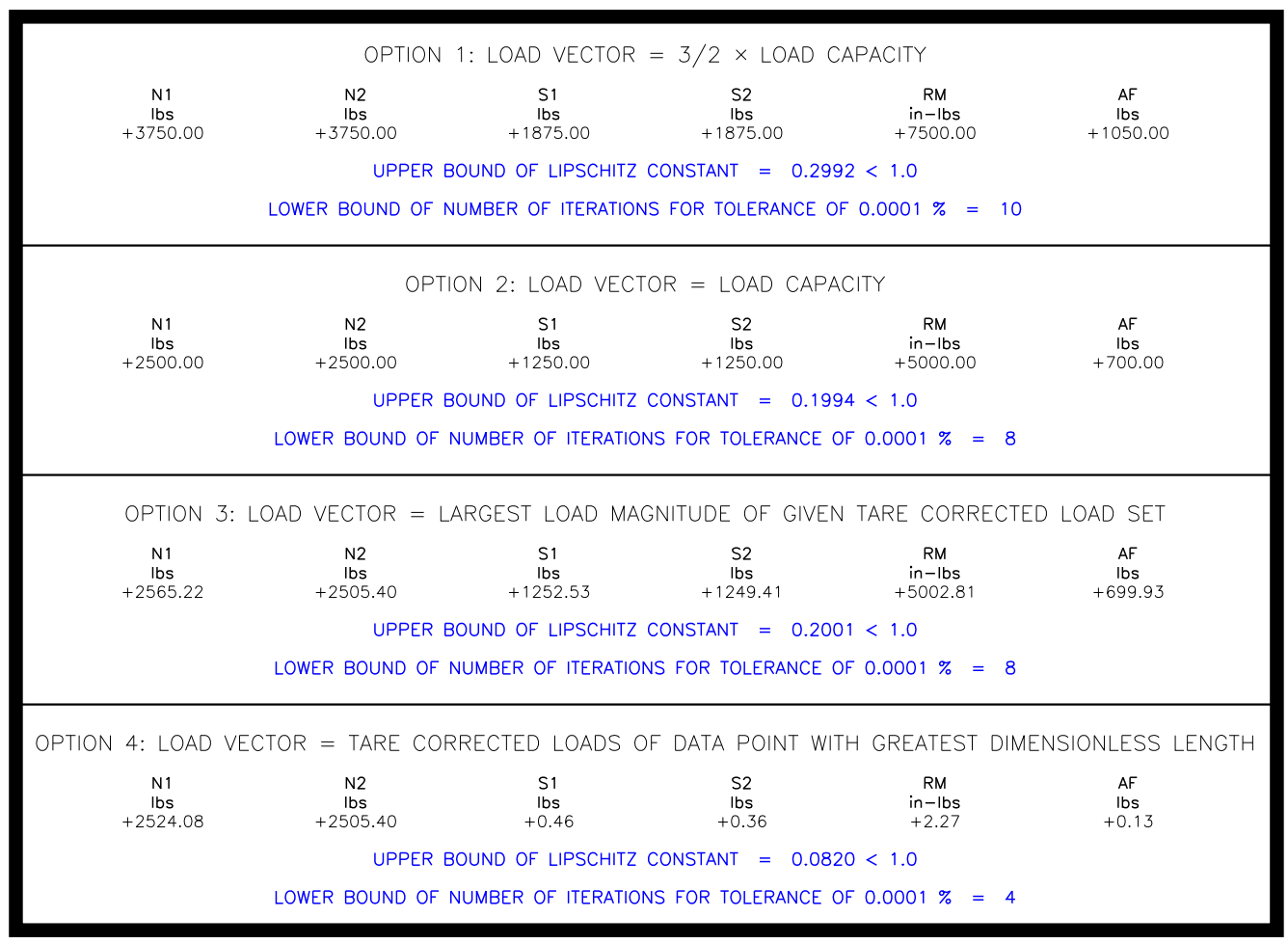

Fig. 1b Convergence test results for the MC60E balance (scaled derivatives, Eqs. (4), (9)).

Again, the upper bound of the Lipschitz constant is well below the threshold of one for all four choices of the upper bound of the load vector. In other words, load iterations are expected to converge in all four selected load regions that the upper bounds of the load vector define.

The second calibration data set came from a manual calibration of NASA's ARC30K five-component semi-span balance. The balance is a direct-read balance that measures two forces and three moment (NF, AF, PM, YM, RM). Table 3 below lists load capacities of the balance:

Table 3: Load capacities of the five-component ARC30K semi-span balance.

\begin{tabular}{|c|c|c|c|c|c|}
\hline & NF, lbs & PM, in-lbs & YM, in-lbs & RM, in-lbs & AF, lbs \\
\hline \hline Capacity & 30000 & 300000 & 150000 & 1300000 & 3000 \\
\hline
\end{tabular}

The balance was calibrated in 2013 in the Large Load Rig that Calspan San Diego uses for the manual calibration of high capacity semi-span balances. The final data set consisted of 1459 points that were distributed across 108 load series. The calibration data was analyzed using iteration equation Type 1 in combination with an optimized math model for each gage output so that large near-linear dependencies could be avoided in the resulting regression models.

Again, the original definition of the iteration convergence test was applied by using unscaled derivatives for the calculation of the Lipschitz constant so that results for the convergence test described in NASA TN D-6860 could be generated. Figure 2a below shows corresponding test results for the four choices of the upper bound of the load vector. In all cases, the upper bound of the Lipschitz constant is well above the threshold of one. This result should indicate severe load iteration convergence problems of the iteration equation that was obtained from the calibration data. However, a closer examination of the actual calibration data analysis results showed that the load iterations converged rapidly. Only two iterations were needed to achieve convergence. This "paradox" between "predicted" and "observed" convergence characteristics can be explained by the fact that (i) unscaled derivatives were used for the calculation of the Lipschitz constant and that (ii) numerical differences between the magnitudes of the load capacities of the ARC30K balance can be very large (from 3000 lbs to 1300000 in-lbs).

American Institute of Aeronautics and Astronautics 


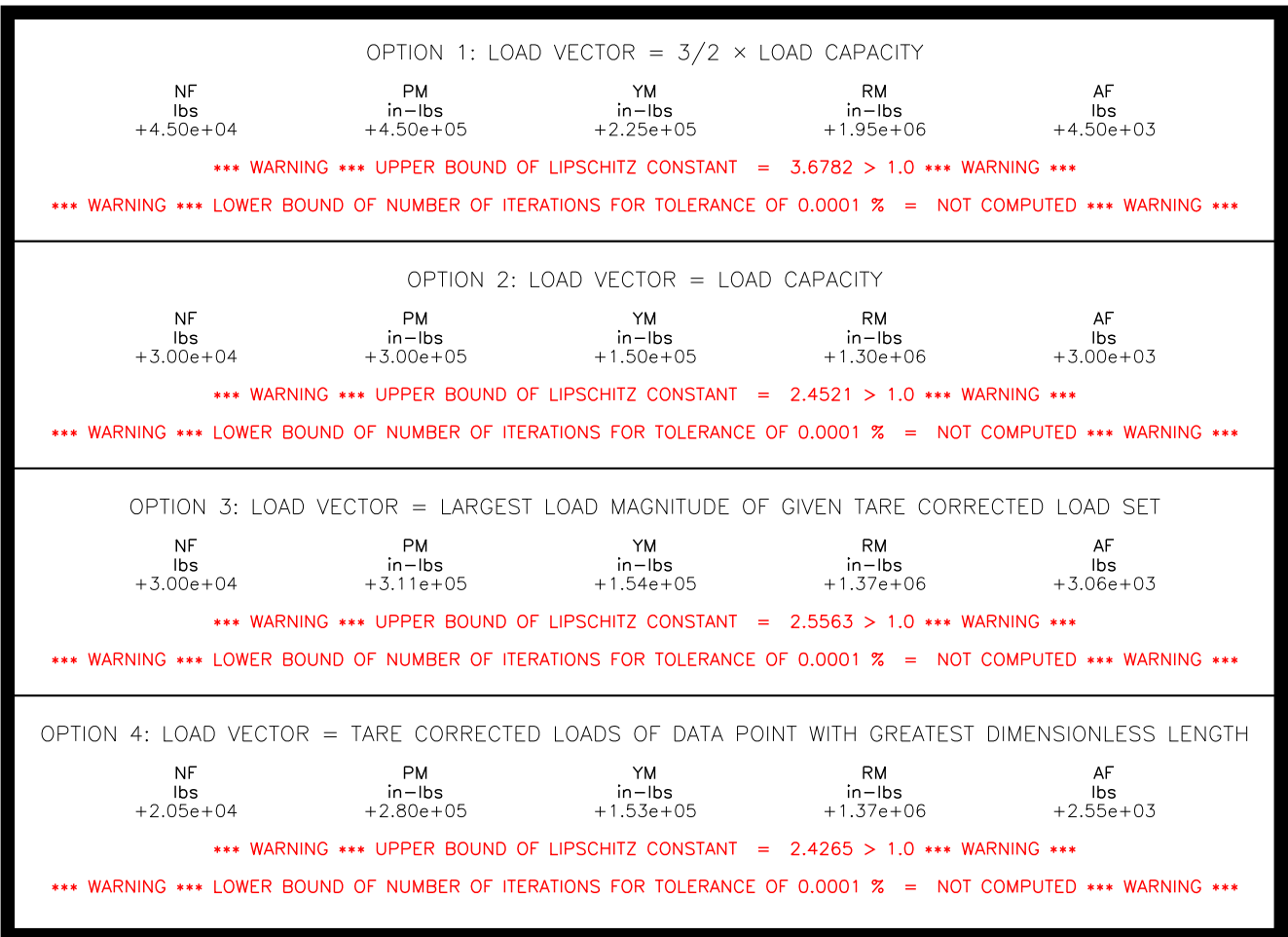

Fig. 2a Convergence test results for the ARC30K balance ( $\underline{\text { unscaled }}$ derivatives, NASA TN D-6860).

Finally, the iteration convergence test was applied by using scaled derivatives for the calculation of the Lipschitz constant. This choice matches the improved iteration convergence test that is defined in Eqs. (4)

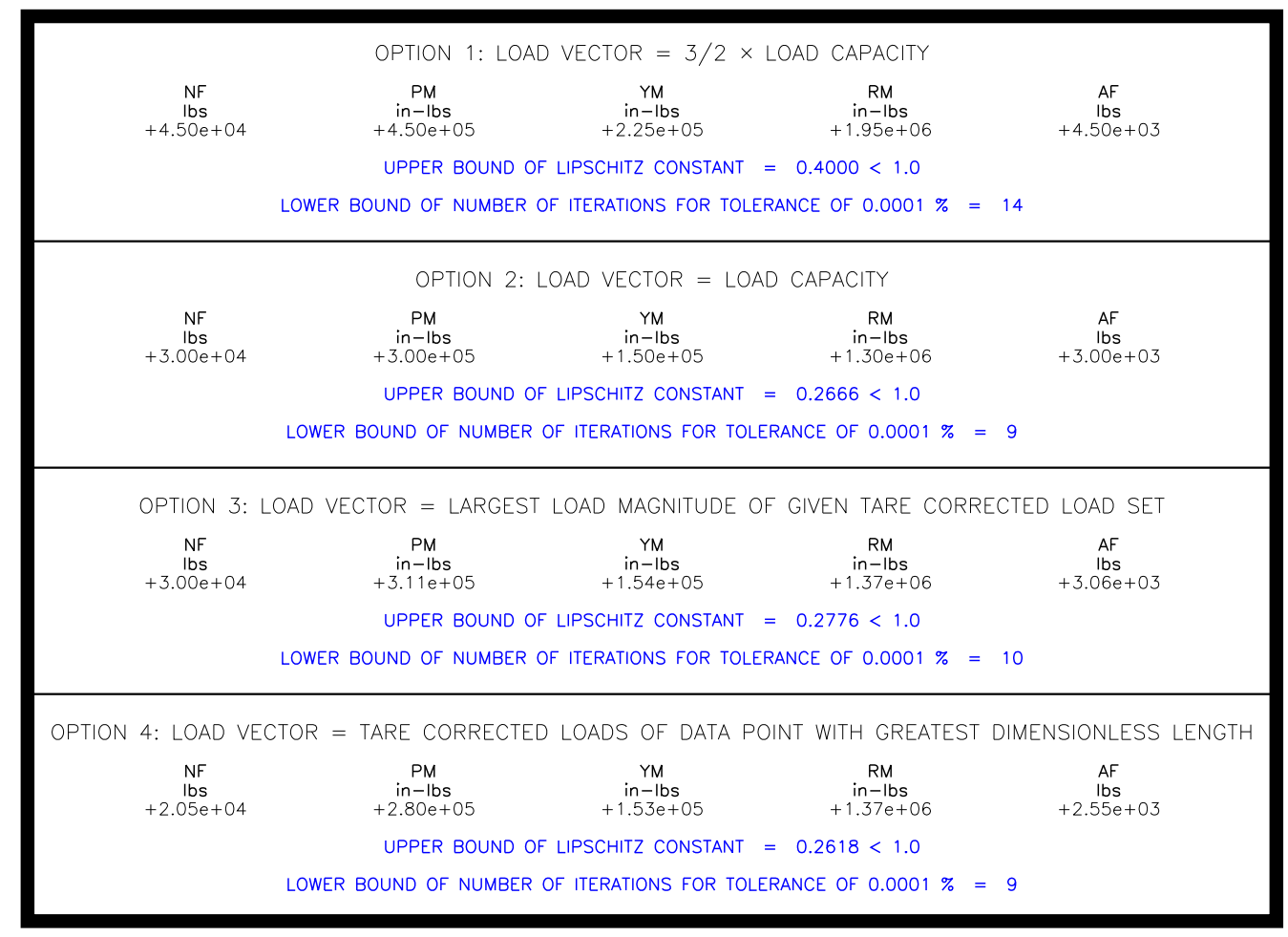

Fig. 2b Convergence test results for the ARC30K balance (scaled derivatives, Eqs. (4), (9)). 
and (9) of the current paper. Figure $2 \mathrm{~b}$ above shows results of this situation for the four choices of the upper bound of the load vector. Now, as expected, the Lipschitz constant is well below the threshold of one for all four cases and load iterations are expected to converge in all four selected load regions. This is the correct convergence test result. It agrees with the rapid iteration convergence characteristics that were actually observed for the balance calibration data of the ARC30K balance.

\section{Summary}

An improved convergence test for iterative strain-gage balance load predictions was developed in order to correct a significant analytical error that is hidden in NASA TN D-6860's original definition of the test. The error leads to a faulty assessment of load iteration convergence characteristics whenever large differences in the magnitudes of strain-gage balance load capacities exist. The author corrected the error by applying a load capacity dependent scale factor such that the numerical inputs for the convergence test are always dimensionless. Calibration data from two wind tunnel balances was used to illustrate benefits of the improved load iteration convergence test for strain-gage balance data.

The author realizes, of course, that only a handful of analysts in the aerospace testing community use the Lipschitz constant for an assessment of the convergence characteristics of wind tunnel balance load iterations. However, the detection and correction of the hidden analytical error in NASA TN D-6860 goes beyond its specific application to balance load iterations. It highlights the fact that the application of abstract concepts from mathematics to real-world problems must be done with great care. In our case, complexities associated with the implementation of the Lipschitz constant simply hid the fundamental facts that (i) the Lipschitz constant is, by definition, a dimensionless quantity and that (ii) all physical variables needed for the calculation of its upper bound must be made dimensionless before use.

\section{Acknowledgements}

The author wants to thank Tom Volden of Jacobs Technology and Bob Gisler of NASA Ames Research Center for their critical and constructive review of the final manuscript. The work reported in this paper was supported by the Wind Tunnel Division at NASA Ames Research Center under contract NNA16BD26C.

\section{References}

[1] Ulbrich, N., "Implementation and Use of a Convergence Test for Iterative Wind Tunnel Balance Load Predictions," AIAA 2018-4109, paper presented at the 2018 AIAA Aerodynamic Measurement Technology and Ground Testing Conference, Atlanta, Georgia, June 2018.

[2] AIAA/GTTC Internal Balance Technology Working Group, Recommended Practice - Calibration and Use of Internal Strain-Gage Balances with Application to Wind Tunnel Testing, AIAA R-091-2003, American Institute of Aeronautics and Astronautics, Reston, Virginia, 2003.

[3] Ulbrich, N., and Volden, T., "Strain-Gage Balance Calibration Analysis Using Automatically Selected Math Models," AIAA 2005-4084, paper presented at the 41st AIAA/ASME/SAE/ASEE Joint Propulsion Conference, Tucson, Arizona, July 2005.

[4] Ulbrich, N., and Volden, T., "Application Of A New Calibration Analysis Process to the MK-III-C Balance," AIAA 2006-0517, paper presented at the 44th AIAA Aerospace Sciences Meeting, Reno, Nevada, January 2006.

[5] Smith, D. L., An Efficient Algorithm Using Matrix Methods to Solve Wind-Tunnel Force-Balance Equations, NASA Technical Note TN D-6860, Langley Research Center, Hampton, Virginia, August 1972.

[6] Henrici, P., Elements of Numerical Analysis, 1st ed., John Wiley \& Sons, Inc., New York, London, Sydney, 1964, pp. 97-105. 